\title{
EFEITOS GÊNICOS E OSCILAÇÃO GENÉTICA ASSOCIADOS À SELEÇÃO RECORRENTE INTRAPOPULACIONAL NA POPULAÇÃO DE MILHO SA3'
}

\author{
MARIO CAVIEDES CEPEDA², CLÁUDIO LOPES DE SOUZA JUNIOR ${ }^{3}$, SHIVAJI PANDEY $^{4}$ e LUIS NARRO LEON ${ }^{5}$
}

\begin{abstract}
RESUMO - Foram avaliados os efeitos da seleção recorrente intrapopulacional e da oscilação genética na população de milho (Zea mays L.) SA3 tolerante a solos ácidos, submetida a 20 ciclos de seleção. Utilizaram-se amostras de oito ciclos seletivos, com o objetivo de estimar as respostas à seleção para produção de grãos e outros caracteres agronômicos, utilizando o modelo proposto por Smith (1983). O material genético utilizado consistiu em oito ciclos per se, oito ciclos per se autofecundados, $28 \mathrm{~F}_{1}$ 's entre ciclos, $28 \mathrm{~F}_{1}$ 's entre ciclos autofecundados e $28 \mathrm{~F}_{1}$ 's recombinados. Os experimentos foram conduzidos em cinco ambientes com solos ácidos, no Brasil e na Colômbia. As magnitudes das respostas esperadas à seleção (2ALI+2DLI) mostraram que os esquemas seletivos utilizados não foram eficientes para melhorar o caráter produção de grãos e para reduzir o caráter altura da planta. Porém, as estimativas indicaram que o processo de seleção foi eficiente para reduzir o número de dias para o florescimento feminino e aumentar o número de espigas por planta. As magnitudes das estimativas da oscilação genética (DQI) mostraram que seus efeitos não limitaram o melhoramento dos diferentes caracteres.
\end{abstract}

Termos para indexação: Zea mays, solos ácidos, características agronômicas, grãos, produção, variação genética, resposta à seleção.

\section{GENIC AND GENETIC DRIFT EFFECTS ASSOCIATED WITH INTRAPOPULATION RECURRENT SELECTION IN THE SA3 MAIZE POPULATION}

\begin{abstract}
The effects of intrapopulation recurrent selection and of the genetic drift were evaluated on 20 cycles of selection for SA3 maize (Zea mays L.) population, tolerant to acid soil conditions. A sample of eight cycles was used to estimate the response to selection for grain yield and other agronomic traits by the model proposed by Smith (1983). The genetic materials used included eight cycles per se, eight cycles per se selfed, $28 \mathrm{~F}_{1}$ 's among cycles, $28 \mathrm{~F}_{1}$ 's among cycles selfed and $28 \mathrm{~F}_{1}$ 's recombined. The trials were evaluated at five environments under acid soils conditions in Brazil and Colombia. The estimates of response to selection $(2 \mathrm{ALI}+2 \mathrm{DLI})$ showed that selection process was not effective to improve grain yield and to reduce plant height. However, the selection procedure was efficient to reduce days to silk and to increase the number of ears per plant. The magnitude of genetic drift estimates (DQI) indicated that its effects did not limit the improvement of the characters evaluated.
\end{abstract}

Index terms: Zea mays, acid soils, agronomic characters, grains, production, genetic variation, selection responses.

\footnotetext{
${ }^{1}$ Aceito para publicação em 10 de janeiro de 2000.

${ }^{2}$ Eng. Agrôn., M.Sc, Instituto Nacional de Investigaciones Agropecuarias (INIAP), Apartado Aéreo 1701-340, Quito, Ecuador. Bolsista da FAPESP.

E-mail: caviedes@dinfor.ecuanex.net.ec

${ }^{3}$ Eng. Agrôn., Ph.D., Dep. de Genética, Escola Superior de Agricultura «Luiz de Queiroz» (ESALQ), Universidade de São Paulo (USP), Caixa Postal, 83, CEP 13418-900 Piracicaba, SP. E-mail: clsouza@carpa.ciagri.usp.br

${ }^{4}$ Eng. Agrôn., Ph.D., Centro Internacional de Mejoramiento de Maíz y Trigo (CIMMYT), Apartado Postal 6-641, C.P. 06600, México, D.F, México. E-mail: spandey@cgiar.org

${ }^{5}$ Eng. Agrôn., Ph.D., CIMMYT, Apartado Aéreo 6713, Cali, Colômbia. E-mail: lnarro@cgiar.org
}

\section{INTRODUÇÃO}

A ocorrência de solos ácidos nos trópicos é bastante comum, e a acidez está associada, em muitos casos à baixa fertilidade desses solos. No Brasil, o problema ocorre em grandes áreas de seu território, expressando-se, em parte, nos solos sob vegetação de cerrado que ocupam uma área de 204 milhões de hectares, o qual representa $22 \%$ de seu território, onde se produz $25 \%$ da produção de milho, soja e arroz (Lopes et al., 1987; Parentoni et al., 1996). O milho cultivado em solos ácidos apresenta problemas que são causados principalmente pela toxicidade 
de $\mathrm{Al}$, que inibe o desenvolvimento das raízes, resultando em menor absorção de nutrientes e umidade. A existência de variabilidade genética e a herança da tolerância à toxidez de $\mathrm{Al}$ já foi estudada e reportada por vários pesquisadores, merecendo destaque os trabalhos de Lima et al. (1992), Duque-Vargas et al. (1994), Borrero et al. (1995), Parentoni et al. (1995), Resende et al. (1997) e Salazar et al. (1997), entre outros. Esses estudos indicaram ainda que a tolerância a solos ácidos pode ser incrementada com seleção recorrente, com base em avaliações sobre vários ambientes de solos ácidos visando melhorar a produção de grãos e outras características agronômicas.

O melhoramento de populações via seleção recorrente tem como objetivo aumentar gradualmente a freqüência dos alelos favoráveis, por meio de ciclos sucessivos de seleção e recombinação dos genótipos superiores, mantendo a variabilidade genética da população (Souza Júnior, 1983). A seleção e a oscilação genética são dois processos que podem alterar as freqüências gênicas e conseqüentemente alterar a magnitude da variância genética de uma população sob seleção recorrente (Helms et al., 1989). Para a análise das mudanças genéticas em uma população submetida à seleção recorrente e avaliar o progresso através de ciclos seletivos, Smith (1979a, 1979b, 1983) apresentou um modelo que permite interpretar as mudanças na média da população sob seleção como função das alterações das freqüências alélicas e dos efeitos alélicos nas populações. O modelo possibilita, também, a mensuração dos efeitos da oscilação genética nas populações selecionadas e a comparação da resposta direta e indireta à seleção.

A avaliação de múltiplos ciclos com diferentes processos de seleção recorrente em milho, utilizando o modelo proposto por Smith, tem sido realizada por vários pesquisadores, cabendo ressaltar os trabalhos de Iglesias \& Hallauer (1989), Keeratinijakal \& Lamkey (1993a, 1993b), Stojsin \& Kannenberg (1994a, 1994b) e Rezende (1997), entre outros. Por outro lado, programas de seleção recorrente incluindo avaliações de progênies e estimativas de progresso por seleção recorrente em solos ácidos são escassos, merecendo destaque os trabalhos de Aguiar (1986), Granados et al. (1993), Pandey et al. (1995) e Resende et al. (1997).

Pesq. agropec. bras., Brasília, v.35, n.8, p.1585-1593, ago. 2000
Com base nesses antecedentes, os objetivos deste trabalho foram estimar a resposta à seleção para produção de grãos e outros caracteres agronômicos na população SA3, estimar as alterações decorrentes dos efeitos de homozigose e heterozigose, e os efeitos da oscilação genética.

\section{MATERIAL E MÉTODOS}

Foram utilizados oito ciclos seletivos (ciclos 2, 5, 8, $11,14,16,18$ e 20) da população de milho SA3, tolerante a solos ácidos, formada pelo Centro Internacional de Mejoramiento de Maíz y Trigo (CIMMYT), a partir de germoplasma do México, da Colômbia, do Peru, da Bolívia, da Tailândia e 14 coleções da raça cateto do Brasil. A população SA3 possui grãos amarelos, semidentados e foi submetida a 16 ciclos de seleção utilizando uma modificação do método "espiga por fileira modificado" com progênies de meios-irmãos (MIM) e quatro ciclos de irmãos germanos (IG) com um total de 20 ciclos de seleção (Granados et al., 1993). No segundo semestre de 1994 e primeiro de 1995 incrementaram-se as sementes dos ciclos per se. A seguir, estes foram autofecundados e cruzados em um sistema dialélico. Posteriormente, esses cruzamentos $F_{1}$ 's foram autofecundados e recombinados.

Os 100 tratamentos obtidos correspondem a oito ciclos per se, oito ciclos per se autofecundados, $28 \mathrm{~F}_{1}$ 's entre os ciclos, $28 \mathrm{~F}_{1}$ 's entre ciclos autofecundados, e $28 \mathrm{~F}_{1}$ 's recombinados. Esses tratamentos foram avaliados nos anos agrícolas de 1995/96 e 1996/97, em cinco ambientes, com solos ácidos localizados no Brasil e na Colômbia, utilizando-se o delineamento de látice 10 x 10 com três repetições em cada ambiente. As parcelas constituíram-se de uma linha de $5 \mathrm{~m}$ de comprimento com espaçamento de $0,75 \mathrm{~m}$ entre linhas e $0,50 \mathrm{~m}$ entre plantas dentro de linhas, com duas plantas por cova perfazendo-se 22 plantas por parcela.

Os ambientes com solos ácidos foram: Carimagua (Colômbia), com $63 \%$ de saturação de $\mathrm{Al}, 10 \mathrm{ppm}$ de $\mathrm{P}$ e pH 4,1; Santander de Quilichao (Colômbia), com 34\% de saturação de $\mathrm{Al}, 10$ ppm de $\mathrm{Pe} \mathrm{pH} \mathrm{4,0;} \mathrm{Villavicencio} 1$ e 2 (Colômbia) com 53\% e 64\% de saturação de Al, 10 ppm de $\mathrm{P}, \mathrm{pH} 4,5$ e 4,3, respectivamente; e Sete Lagoas, MG (Brasil), com 36\% de saturação de Al, 5 ppm de P e pH 5,1.

Foram avaliados e analisados os seguintes caracteres: dias para florescimento feminino, altura da planta (média de cinco plantas por parcela), número de espigas por planta (número de espigas/número de plantas por parcela) e produção de grãos. O caráter produção de grãos foi avaliado através do peso total de espigas despalhadas, assumin- 
do que $80 \%$ desse peso corresponde ao peso de grãos, o qual foi ajustado para $15 \%$ de umidade e corrigido para estande médio (Steel \& Torrie, 1960).

A análise de variância foi realizada quanto a cada caráter em cada ambiente e conjunta dos cinco ambientes. Os efeitos de tratamentos foram considerados como fixos, e os ambientes, como aleatórios. As análises individuais e conjuntas foram realizadas segundo o delineamento em látice de 10 x 10, com recuperação da informação intrabloco (Cochran \& Cox, 1957; Pimentel-Gomes \& Garcia, 1991). No cálculo da análise conjunta, utilizaram-se os totais ajustados de tratamentos estimados nas análises individuais.

Progressos com a seleção foram estimados considerando-se em conjunto os oito ciclos seletivos correspondentes à seleção combinada de meios-irmãos modificados e irmãos germanos (MIM-IG), sobre os ciclos MIM (2, 5, $8,11,14$ e 16$)$ e sobre os ciclos de IG $(16,18$ e 20$)$. O ciclo 16 de MIM foi considerado o ciclo zero $\left(\mathrm{C}_{0}\right)$ de IG (Granados et al., 1993).

As estimativas das alterações nas médias resultantes da seleção foram obtidas pela análise de regressão pelo modelo sugerido por Eberhart (1964). As alterações nos efeitos genéticos resultantes da seleção e da oscilação genética foram estimadas segundo o modelo genético proposto por Smith (1979a, 1979b, 1983). Na obtenção dessas estimativas, utilizaram-se as médias ajustadas de tratamentos das análises conjuntas.

De acordo com o modelo proposto por Smith, $\mathrm{C}_{\mathrm{ij}}$ representa a média da população $\mathrm{i}$, com $\mathrm{j}$ ciclos de seleção. As médias das populações per se, per se autofecundadas, os $F_{1}$ 's entre ciclos, os $F_{1}$ 's autofecundados e os $F_{1}$ 's recombinados podem ser expressos da seguinte forma:

$\mathrm{C}_{\mathrm{ij}}=\mathrm{AOI}+2 \mathrm{DOI}+(\mathrm{J}) 2 \mathrm{ALI}+(\mathrm{J}) 2 \mathrm{DLI}+(\mathrm{J})^{2} 2 \mathrm{DQI}$;

$\mathrm{C}_{\mathrm{ij}}$ autofecundada $=\mathrm{AOI}+\mathrm{DOI}+(\mathrm{J}) 2 \mathrm{ALI}+(\mathrm{J}) \mathrm{DLI}+(\mathrm{J})^{2} \mathrm{DQI}$;

$\left(\mathrm{C}_{\mathrm{ij}} \mathrm{x} \mathrm{C}_{\mathrm{ij}}{ }^{\prime}\right) \mathrm{F}_{1}=\mathrm{AOI}+2 \mathrm{DOI}+\left(\mathrm{J}+\mathrm{J}^{\prime}\right) 2 \mathrm{ALI}+\left(\mathrm{J}+\mathrm{J}^{\prime}\right) 2 \mathrm{DLI}+\left(\mathrm{JxJ} \mathrm{J}^{\prime}\right) 2 \mathrm{DQI}$;

$\left(C_{i j} \mathrm{x}_{\mathrm{ij}}{ }^{\prime}\right) \mathrm{F}_{1}$ autofecundada $=$ AOI+DOI+(J+J')2ALI+(J+J')DLI+(JxJ')DQI; $\left(\mathrm{C}_{\mathrm{ij}} \times \mathrm{C}_{\mathrm{ij}}\right) \mathrm{F}_{1}$ recombinada $=\left(\mathrm{C}_{\mathrm{ij}} \times \mathrm{C}_{\mathrm{ij}}\right) \mathrm{F}_{1}$

onde $\mathrm{C}_{\mathrm{ij}} \mathrm{e} \mathrm{C}_{\mathrm{ij}}$ ' correspondem à população i no j-ésimo ciclo seletivo; AOI e DOI referem-se à contribuição dos homozigotos e heterozigotos no ciclo zero da população i respectivamente; ALI e DLI referem-se aos coeficientes de regressão lineares das alterações das frequiências alélicas causadas pelos homozigotos e heterozigotos na população i, respectivamente, e DQI refere-se ao coeficiente de regressão quadrático das alterações nas frequiências alélicas causadas pela oscilação genética na população i. Os parâmetros do modelo foram estimados pela análise de quadrados mínimos, onde $\mathrm{b}=\left(\mathrm{X}^{\prime} \mathrm{X}\right)^{-1} \mathrm{X}^{\prime} \mathrm{Y}$, sendo $\left(\mathrm{X}^{\prime} \mathrm{X}\right)^{-1}$ a inversa da matriz $X^{\prime} X, X^{\prime}$ é a transposta da matriz $X$ e $Y$ matriz de médias dos tratamentos através dos ambientes com solos ácidos. A matriz X é função do número de ciclos e dos coeficientes dos parâmetros.

As variâncias de cada estimativa $\left[\mathrm{V}\left(\mathrm{B}_{\mathrm{i}}\right)\right]$ foram obtidas multiplicando-se a diagonal principal da matriz $\left(\mathrm{X}^{\prime} \mathrm{X}\right)^{-1}$ pela relação entre o quadrado médio do erro da análise da variância conjunta e o produto do número de repetições pelo número de ambientes utilizados nos ensaios. Os erros associados às estimativas $\left[\mathrm{S}\left(\mathrm{B}_{\mathrm{i}}\right)\right]$ foram obtidos pela extração da raiz quadrada da variância de cada estimativa e utilizados para determinar o nível de significância dos parâmetros do modelo. Deve-se indicar também que foram obtidas estimativas dos seguintes parâmetros genéticos: $\mathrm{b}_{\mathrm{o}}=\mathrm{AOI}+2 \mathrm{DOI}$, o qual estima a contribuição da população original; $b_{1}=2 A L I+2 D L I$, o que estima a resposta esperada à seleção; e $b_{q}=2 D Q I$, o qual estima o efeito da oscilação genética. A porcentagem da resposta à seleção foi estimada pela relação entre o coeficiente de regressão linear $\left(b_{1}\right)$ e o intercepto $\left(b_{o}\right)$ e multiplicado por 100 .

\section{RESULTADOS E DISCUSSÃO}

Diferenças altamente significativas $(\mathrm{P} \leq 0,01)$ foram observadas nos caracteres produção de grãos, dias para florescimento feminino, altura da planta e número de espigas por planta. Os coeficientes de variação para produção de grãos e número de espigas por planta apresentaram-se altos $(25,6 \%$ e $18,9 \%$, respectivamente), em comparação com os relatados na literatura de milho por Hallauer \& Miranda Filho (1981), baixos para o número de dias para o florescimento feminino $(3,1 \%)$ e médios para altura da planta (8,3\%) (Scapim et al., 1995). As médias dos quatro caracteres foram: $2,76 \mathrm{tha}^{-1}$ de grãos; 65,1 dias para o florescimento feminino; $164,5 \mathrm{~cm}$ de altura das plantas e 0,91 espigas por planta.

As médias ajustadas de tratamentos, relativas ao caráter produção de grãos, na população SA3 encontram-se na Tabela 1 . O efeito de tratamentos apresentou diferenças altamente significativas $(\mathrm{P} \leq 0,01)$ em todos os caracteres; esse resultado era esperado em razão de que nessa fonte de variação estão embutidos os efeitos de uma amostra de 20 ciclos seletivos da população SA3. A interação tratamentos x ambientes foi significativa $(\mathrm{P} \leq 0,05)$ para número de espigas por planta e altamente significativa $(P \leq 0,01)$ para produção de grãos e altura da planta, evidenciando o desempenho diferencial dos tratamentos atra- 
TABELA 1. Médias ajustadas de tratamentos do caráter produção de grãos (t ha' $\left.\mathbf{~}^{-1}\right)$ na população SA3, avaliada em ambientes de solos ácidos. 1995-19971.

\begin{tabular}{|c|c|c|c|c|c|}
\hline Tratamento & Média & Tratamento & Média & Tratamento & Média \\
\hline $\mathrm{C}_{2}-\mathrm{S}_{0}$ & 5,13 & $\mathrm{C}_{11} \times \mathrm{C}_{14}-\mathrm{S}_{0}$ & 6,44 & $\mathrm{C}_{14} \times \mathrm{C}_{18}-\mathrm{R}$ & 5,94 \\
\hline $\mathrm{C}_{5}-\mathrm{S}_{0}$ & 5,58 & $\mathrm{C}_{11} \times \mathrm{C}_{16}-\mathrm{S}_{0}$ & 6,17 & $\mathrm{C}_{14} \times \mathrm{C}_{20}-\mathrm{R}$ & 5,77 \\
\hline $\mathrm{C}_{8}-\mathrm{S}_{0}$ & 6,06 & $\mathrm{C}_{11} \times \mathrm{C}_{18}-\mathrm{S}_{0}$ & 6,17 & $\mathrm{C}_{16} \times \mathrm{C}_{18}-\mathrm{R}$ & 6,05 \\
\hline $\mathrm{C}_{11}-\mathrm{S}_{0}$ & 5,89 & $\mathrm{C}_{11} \times \mathrm{C}_{20}-\mathrm{S}_{0}$ & 6,13 & $\mathrm{C}_{16} \times \mathrm{C}_{20}-\mathrm{R}$ & 6,11 \\
\hline $\mathrm{C}_{14}-\mathrm{S}_{0}$ & 6,11 & $\mathrm{C}_{14} \times \mathrm{C}_{16}-\mathrm{S}_{0}$ & 5,97 & $\mathrm{C}_{18} \times \mathrm{C}_{20}-\mathrm{R}$ & 6,01 \\
\hline $\mathrm{C}_{16}-\mathrm{S}_{0}$ & 5,28 & $\mathrm{C}_{14} \times \mathrm{C}_{18}-\mathrm{S}_{0}$ & 6,13 & $\mathrm{C}_{2} \times \mathrm{C}_{5}-\mathrm{S}_{1}$ & 2,84 \\
\hline $\mathrm{C}_{18}-\mathrm{S}_{0}$ & 6,17 & $\mathrm{C}_{14} \times \mathrm{C}_{20}-\mathrm{S}_{0}$ & 5,24 & $\mathrm{C}_{2} \times \mathrm{C}_{8}-\mathrm{S}_{1}$ & 2,86 \\
\hline $\mathrm{C}_{20}-\mathrm{S}_{0}$ & 5,64 & $\mathrm{C}_{16} \times \mathrm{C}_{18}-\mathrm{S}_{0}$ & 5,26 & $\mathrm{C}_{2} \times \mathrm{C}_{11}-\mathrm{S}_{1}$ & 2,42 \\
\hline $\mathrm{C}_{2}-\mathrm{S}_{1}$ & 2,56 & $\mathrm{C}_{16} \times \mathrm{C}_{20}-\mathrm{S}_{0}$ & 5,46 & $\mathrm{C}_{2} \times \mathrm{C}_{14}-\mathrm{S}_{1}$ & 2,44 \\
\hline $\mathrm{C}_{5}-\mathrm{S}_{1}$ & 2,99 & $\mathrm{C}_{18} \times \mathrm{C}_{20}-\mathrm{S}_{0}$ & 5,09 & $\mathrm{C}_{2} \times \mathrm{C}_{16}-\mathrm{S}_{1}$ & 3,67 \\
\hline $\mathrm{C}_{8}-\mathrm{S}_{1}$ & 2,95 & $\mathrm{C}_{2} \times \mathrm{C}_{5}-\mathrm{R}$ & 5,80 & $\mathrm{C}_{2} \times \mathrm{C}_{18}-\mathrm{S}_{1}$ & 2,94 \\
\hline $\mathrm{C}_{11}-\mathrm{S}_{1}$ & 2,53 & $\mathrm{C}_{2} \times \mathrm{C}_{8}-\mathrm{R}$ & 5,90 & $\mathrm{C}_{2} \times \mathrm{C}_{20}-\mathrm{S}_{1}$ & 3,38 \\
\hline $\mathrm{C}_{14}-\mathrm{S}_{1}$ & 2,73 & $\mathrm{C}_{2} \times \mathrm{C}_{11}-\mathrm{R}$ & 5,87 & $\mathrm{C}_{5} \times \mathrm{C}_{8}-\mathrm{S}_{1}$ & 2,57 \\
\hline $\mathrm{C}_{16}-\mathrm{S}_{1}$ & 3,13 & $\mathrm{C}_{2} \times \mathrm{C}_{14}-\mathrm{R}$ & 5,92 & $\mathrm{C}_{5} \times \mathrm{C}_{11}-\mathrm{S}_{1}$ & 2,51 \\
\hline $\mathrm{C}_{18}-\mathrm{S}_{1}$ & 3,27 & $\mathrm{C}_{2} \times \mathrm{C}_{16}-\mathrm{R}$ & 5,54 & $\mathrm{C}_{5} \times \mathrm{C}_{14}-\mathrm{S}_{1}$ & 3,29 \\
\hline $\mathrm{C}_{20}-\mathrm{S}_{1}$ & 3,12 & $\mathrm{C}_{2} \times \mathrm{C}_{18}-\mathrm{R}$ & 5,96 & $\mathrm{C}_{5} \times \mathrm{C}_{16}-\mathrm{S}_{1}$ & 2,92 \\
\hline $\mathrm{C}_{2} \times \mathrm{C}_{5}-\mathrm{S}_{0}$ & 5,75 & $\mathrm{C}_{2} \times \mathrm{C}_{20}-\mathrm{R}$ & 6,30 & $\mathrm{C}_{5} \times \mathrm{C}_{18}-\mathrm{S}_{1}$ & 2,77 \\
\hline $\mathrm{C}_{2} \times \mathrm{C}_{8}-\mathrm{S}_{0}$ & 6,14 & $\mathrm{C}_{5} \times \mathrm{C}_{8}-\mathrm{R}$ & 5,92 & $\mathrm{C}_{5} \times \mathrm{C}_{20}-\mathrm{S}_{1}$ & 2,88 \\
\hline $\mathrm{C}_{2} \times \mathrm{C}_{11}-\mathrm{S}_{0}$ & 6,45 & $\mathrm{C}_{5} \times \mathrm{C}_{11}-\mathrm{R}$ & 5,65 & $\mathrm{C}_{8} \times \mathrm{C}_{11}-\mathrm{S}_{1}$ & 2,54 \\
\hline $\mathrm{C}_{2} \times \mathrm{C}_{14}-\mathrm{S}_{0}$ & 5,69 & $\mathrm{C}_{5} \times \mathrm{C}_{14}-\mathrm{R}$ & 5,87 & $\mathrm{C}_{8} \times \mathrm{C}_{14}-\mathrm{S}_{1}$ & 2,97 \\
\hline $\mathrm{C}_{2} \times \mathrm{C}_{16}-\mathrm{S}_{0}$ & 6,59 & $\mathrm{C}_{5} \times \mathrm{C}_{16}-\mathrm{R}$ & 5,72 & $\mathrm{C}_{8} \times \mathrm{C}_{16}-\mathrm{S}_{1}$ & 2,76 \\
\hline $\mathrm{C}_{2} \times \mathrm{C}_{18}-\mathrm{S}_{0}$ & 6,39 & $\mathrm{C}_{5} \times \mathrm{C}_{18}-\mathrm{R}$ & 6,07 & $\mathrm{C}_{8} \times \mathrm{C}_{18}-\mathrm{S}_{1}$ & 2,88 \\
\hline $\mathrm{C}_{2} \times \mathrm{C}_{20}-\mathrm{S}_{0}$ & 6,29 & $\mathrm{C}_{5} \times \mathrm{C}_{20}-\mathrm{R}$ & 5,73 & $\mathrm{C}_{8} \times \mathrm{C}_{20}-\mathrm{S}_{1}$ & 2,85 \\
\hline $\mathrm{C}_{5} \times \mathrm{C}_{8}-\mathrm{S}_{0}$ & 6,12 & $\mathrm{C}_{8} \times \mathrm{C}_{11}-\mathrm{R}$ & 5,59 & $\mathrm{C}_{11} \times \mathrm{C}_{14}-\mathrm{S}_{1}$ & 2,61 \\
\hline $\mathrm{C}_{5} \times \mathrm{C}_{11}-\mathrm{S}_{0}$ & 5,88 & $\mathrm{C}_{8} \times \mathrm{C}_{14}-\mathrm{R}$ & 5,72 & $\mathrm{C}_{11} \times \mathrm{C}_{16}-\mathrm{S}_{1}$ & 2,37 \\
\hline $\mathrm{C}_{5} \times \mathrm{C}_{14}-\mathrm{S}_{0}$ & 5,35 & $\mathrm{C}_{8} \times \mathrm{C}_{16}-\mathrm{R}$ & 5,63 & $\mathrm{C}_{11} \times \mathrm{C}_{18}-\mathrm{S}_{1}$ & 2,85 \\
\hline $\mathrm{C}_{5} \times \mathrm{C}_{16}-\mathrm{S}_{0}$ & 5,75 & $\mathrm{C}_{8} \times \mathrm{C}_{18}-\mathrm{R}$ & 6,19 & $\mathrm{C}_{11} \times \mathrm{C}_{20}-\mathrm{S}_{1}$ & 2,71 \\
\hline $\mathrm{C}_{5} \times \mathrm{C}_{18}-\mathrm{S}_{0}$ & 5,43 & $\mathrm{C}_{8} \times \mathrm{C}_{20}-\mathrm{R}$ & 6,04 & $\mathrm{C}_{14} \times \mathrm{C}_{16}-\mathrm{S}_{1}$ & 2,69 \\
\hline $\mathrm{C}_{5} \times \mathrm{C}_{20}-\mathrm{S}_{0}$ & 4,99 & $\mathrm{C}_{11} \times \mathrm{C}_{14}-\mathrm{R}$ & 6,22 & $\mathrm{C}_{14} \times \mathrm{C}_{18}-\mathrm{S}_{1}$ & 2,87 \\
\hline $\mathrm{C}_{8} \times \mathrm{C}_{11}-\mathrm{S}_{0}$ & 6,36 & $\mathrm{C}_{11} \times \mathrm{C}_{16}-\mathrm{R}$ & 6,28 & $\mathrm{C}_{14} \times \mathrm{C}_{20}-\mathrm{S}_{1}$ & 2,97 \\
\hline $\mathrm{C}_{8} \times \mathrm{C}_{14}-\mathrm{S}_{0}$ & 6,17 & $\mathrm{C}_{11} \times \mathrm{C}_{18}-\mathrm{R}$ & 6,18 & $\mathrm{C}_{16} \times \mathrm{C}_{18}-\mathrm{S}_{1}$ & 2,87 \\
\hline $\mathrm{C}_{8} \times \mathrm{C}_{16}-\mathrm{S}_{0}$ & 6,14 & $\mathrm{C}_{11} \times \mathrm{C}_{20}-\mathrm{R}$ & 5,83 & $\mathrm{C}_{16} \times \mathrm{C}_{20}-\mathrm{S}_{1}$ & 3,05 \\
\hline $\mathrm{C}_{8} \times \mathrm{C}_{18}-\mathrm{S}_{0}$ & 6,13 & $\mathrm{C}_{14} \times \mathrm{C}_{16}-\mathrm{R}$ & 6,06 & $\mathrm{C}_{18} \times \mathrm{C}_{20}-\mathrm{S}_{1}$ & 2,81 \\
\hline $\mathrm{C}_{8} \times \mathrm{C}_{20}-\mathrm{S}_{0}$ & 6,31 & & & & \\
\hline
\end{tabular}

${ }^{1} \mathrm{~S}_{0}, \mathrm{~S}_{1}$ e R referem-se às gerações não-endogâmicas, com uma geração de autofecundação e com uma geração de recombinação, respectivamente; média geral $=2,76 \mathrm{t} \mathrm{ha}^{-1}$; coeficiente de variação $=25,6 \%$.

vés dos ambientes com solos ácidos, e não-significativa no caráter dias para o florescimento feminino, sugerindo que não houve variação dos ciclos seletivos em função das mudanças ambientais.

Em seu conjunto, esses resultados indicam que ambientes com altos níveis de $\mathrm{Al}$ tóxico reduzem significativamente a produção de grãos, a altura da planta e o número de espigas por planta e retarda o florescimento do milho. Resultados semelhantes já foram relatados em outros trabalhos realizados com diferentes populações de milho sob condições de estresse por Al (Aguiar, 1986; Duque-Vargas et al., 1994; Borrero et al., 1995; Resende et al., 1997).

Em relação às estimativas de parâmetros genéticos do caráter produção de grãos, a contribuição dos efeitos aditivos (AOI) na população original SA3 não apresentou significância nos três processos seletivos, sendo negativa e de baixa magnitude $\left(-0,04 \mathrm{t} \mathrm{ha}^{-1}\right)$ no processo de seleção combinada de meios-irmãos modificados e irmãos germanos, e positivas e de maior magnitude $\left(0,34 \mathrm{tha}^{-1}\right.$ e $\left.0,25 \mathrm{tha}^{-1}\right)$ nos processos de meios-irmãos modificados e de ir- 
mãos germanos, respectivamente. Os efeitos de dominância (DOI) na população original foram positivos $\left(1,60 \mathrm{t} \mathrm{ha}^{-1}, 1,28 \mathrm{t} \mathrm{ha}^{-1}\right.$ e 1,50 $\left.\mathrm{t} \mathrm{ha}^{-1}\right)$ e significativos $(\mathrm{P} \leq 0,01)$ nos três processos seletivos, evidenciando o pronunciado efeito causado pela dominância dos alelos que controlam produção de grãos na população SA3 (Tabela 2).

Os efeitos aditivos (ALI) e de dominância (DLI) decorrentes da seleção para produção de grãos foram positivos $\left(0,26 \times 10^{-2} \mathrm{t} \mathrm{ha}^{-1}\right.$ e $0,10 \times 10^{-2} \mathrm{t} \mathrm{ha}^{-1}$, respectivamente) e não-significativos na seleção combinada. Entretanto, no processo de meios-irmãos modificado, os efeitos aditivos foram negativos $\left(-0,11 \times 10^{-1} \mathrm{tha}^{-1}\right)$ e significativos $(\mathrm{P} \leq 0,10)$, e negativos $\left(-0,05 \times 10^{-1} \mathrm{t} \mathrm{ha}^{-1}\right)$ e não-significativos em irmãos germanos. Por outro lado, as estimativas dos efeitos de dominância foram positivas $\left(0,22 \times 10^{-1} \mathrm{t} \mathrm{ha}^{-1}\right.$ e $\left.0,19 \times 10^{-1} \mathrm{tha}^{-1}\right)$ nesses dois processos, sendo significativa $(\mathrm{P} \leq 0,01)$ no caso de meios-irmãos modificados e não-significativas para irmãos germanos. Esses resultados evidenciam que ocorreram aumentos nas freqüências dos alelos favoráveis nos locos com algum nível de dominância, e portanto a contribuição dos efeitos de dominância foram superiores aos efeitos aditivos para o melhoramento da população SA3. Os efeitos da oscilação genética (DQI) para os três processos seletivos foram positivos, não-significativos e com baixas magnitudes $\left(0,11 \times 10^{-3} \mathrm{tha}^{-1}, 0,04 \times 10^{-2} \mathrm{t} \mathrm{ha}^{-1} \mathrm{e}\right.$ $0,08 \times 10^{-1} \mathrm{t} \mathrm{ha}^{-1}$, respectivamente). Esses resultados indicam que os efeitos da oscilação genética não foram importantes na população melhorada, porém direcionais, apresentando tendência de aumentar a produção de grãos (Tabela 2).

TABELA 2. Estimativas dos efeitos aditivos (AOI) e de dominância (DOI) na população original; dos efeitos aditivos (ALI) e de dominância (DLI) causados pela seleção e dos efeitos de oscilação genética (DQI) na população SA3, em três processos seletivos e quatro caracteres agronômicos em ambientes com solos ácidos (1995-1997).

\begin{tabular}{|c|c|c|c|c|c|}
\hline $\begin{array}{l}\text { Processo } \\
\text { seletivo }^{1}\end{array}$ & Parâmetro & $\begin{array}{l}\text { Produção de grãos } \\
\left(\mathrm{t} \mathrm{ha}^{-1}\right)\end{array}$ & $\begin{array}{l}\text { Florescimento feminino } \\
\text { (dias) }\end{array}$ & $\begin{array}{l}\text { Altura da planta } \\
\qquad(\mathrm{cm})\end{array}$ & $\begin{array}{c}\text { Número de espigas } \\
\text { por planta }\end{array}$ \\
\hline \multirow[t]{6}{*}{ MIM-IG } & AOI & $-0,04^{\mathrm{ns}} \pm 0,17$ & $67,79 * * \pm 0,48$ & $138,82^{* *} \pm 3,26$ & $0,59 * * \pm 0,04$ \\
\hline & DOI & $1,60 * * \pm 0,10$ & $-1,76^{* *} \pm 0,29$ & $14,75 * * \pm 1,97$ & $0,17^{* *} \pm 0,02$ \\
\hline & $\mathrm{ALI}^{2}$ & $0,26^{\mathrm{ns}} \pm 0,37$ & $0,52^{\mathrm{ns}} \pm 1,04$ & $13,39 * * \pm 7,13$ & $0,09^{\mathrm{ns}} \pm 0,09$ \\
\hline & $\mathrm{DLI}^{2}$ & $0,10^{\mathrm{ns}} \pm 0,46$ & $-3,06 * \pm 1,31$ & $-12,07^{\mathrm{ns}} \pm 8,98$ & $-0,008^{\mathrm{ns}} \pm 0,11$ \\
\hline & $\mathrm{DQI}^{3}$ & $0,11^{\mathrm{ns}} \pm 0,15$ & $0,61^{\mathrm{ns}} \pm 0,43$ & $0,26^{\mathrm{ns}} \pm 2,97$ & $0,020^{\mathrm{ns}} \pm 0,037$ \\
\hline & $\mathrm{R}^{2}(\%)$ & 86,77 & 68,16 & 63,67 & 75,28 \\
\hline \multirow[t]{6}{*}{ MIM } & AOI & $0,34^{\mathrm{ns}} \pm 0,21$ & $67,78 * * \pm 0,60$ & $139,69 * * \pm 5,41$ & $0,57^{* *} \pm 0,05$ \\
\hline & DOI & $1,28 * * \pm 0,13$ & $-1,11 * * \pm 0,36$ & $13,83^{* *} \pm 3,27$ & $0,18 * * \pm 0,03$ \\
\hline & $\mathrm{ALI}^{4}$ & $-0,11 * * * \pm 0,06$ & $0,48 * * \pm 0,17$ & $0,97^{\mathrm{ns}} \pm 1,53$ & $0,01^{\mathrm{ns}} \pm 0,01$ \\
\hline & $\mathrm{DLI}^{4}$ & $0,22 * * \pm 0,07$ & $-0,92 * * \pm 0,21$ & $-0,84^{\mathrm{ns}} \pm 1,90$ & $-0,008^{\mathrm{ns}} \pm 0,020$ \\
\hline & $\mathrm{DQI}^{2}$ & $0,04^{\mathrm{ns}} \pm 0,03$ & $0,32 * * \pm 0,08$ & $0,92^{\mathrm{ns}} \pm 0,73$ & $0,0016^{\mathrm{ns}} \pm 0,0070$ \\
\hline & $\mathrm{R}^{2}(\%)$ & 90,90 & 74,69 & 68,92 & 80,21 \\
\hline \multirow[t]{6}{*}{ IG } & $\mathrm{AOI}$ & $0,25^{\mathrm{ns}} \pm 0,30$ & $69,14 * * \pm 0,86$ & $150,68 * * \pm 5,90$ & $0,54^{\mathrm{ns}} \pm 0,07$ \\
\hline & DOI & $1,50 * * \pm 0,19$ & $-1,99 * * \pm 0,52$ & $10,08 * * \pm 3,61$ & $0,20 * * \pm 0,04$ \\
\hline & $\mathrm{ALI}^{4}$ & $-0,05^{\mathrm{ns}} \pm 0,42$ & $-0,52^{\mathrm{ns}} \pm 1,19$ & $-6,16^{\mathrm{ns}} \pm 8,19$ & $0,21^{* * *} \pm 0,10$ \\
\hline & $\mathrm{DLI}^{4}$ & $0,19^{\mathrm{ns}} \pm 0,51$ & $-0,64^{\mathrm{ns}} \pm 1,45$ & $6,37^{\mathrm{ns}} \pm 9,96$ & $-0,12^{\mathrm{ns}} \pm 0,12$ \\
\hline & $\mathrm{DQI}^{4}$ & $0,08^{\mathrm{ns}} \pm 0,06$ & $-0,37 * * \pm 0,17$ & $-2,24 * * * \pm 1,19$ & $0,003^{\mathrm{ns}} \pm 0,015$ \\
\hline & $\mathrm{R}^{2}(\%)$ & 93,93 & 78,42 & 82,29 & 74,22 \\
\hline
\end{tabular}


A resposta esperada à seleção segundo o modelo de Smith (1983) é definida como (2ALI + 2DLI), e é uma função da resposta observada (2ALI + 2DLI + 2DQI) corrigida pelos efeitos da oscilação genética. Como os efeitos da oscilação genética (DQI) não foram significativos, não afetaram a resposta à seleção. Esses resultados eram esperados, uma vez que foram utilizadas em média 25 progênies de meiosirmãos ou de irmãos germanos para a recombinação, as quais geram populações melhoradas com elevado tamanho efetivo populacional. A resposta foi de $0,72 \times 10^{-2} \mathrm{t} \mathrm{ha}^{-1}$ por ciclo $(0,23 \%)$ para seleção combinada; de $0,22 \times 10^{-2} \mathrm{t} \mathrm{ha}^{-1}$ por ciclo $(0,72 \%)$ para meios-irmãos modificados, e de $0,28 \times 10^{-1} \mathrm{tha}^{-1}$ por ciclo $(0,89 \%)$ para irmãos germanos (Tabela 3$)$. As estimativas obtidas nesta pesquisa são bastante inferiores às obtidas com a mesma população por Granados et al. (1993) e Pandey et al. (1995). Também, são bastante inferiores às estimativas reportadas por outros autores em avaliações realizadas com diferentes populações de milho sob condições de solos ácidos (Aguiar, 1986; Lima et al., 1992; Resende et al., 1997).

Quanto ao florescimento feminino, a estimativa do parâmetro genético (AOI) na população original SA3, nos três processos seletivos, apresentou contribuição positiva e significativa $(P \leq 0,01)$, com magnitudes de 67,79 dias na seleção combinada, 67,78 dias no processo de meios-irmãos modificados e 69,41 dias no de irmãos germanos. Entretanto, a contribuição dos efeitos de dominância (DOI) na população original foi negativa, significativa $(\mathrm{P} \leq 0,01)$, e de menor magnitude nos três processos (1,76 dias,
$-1,11$ dias e -1,99 dias, respectivamente). Tais resultados sugerem que o caráter dias para florescimento feminino foi fortemente controlado por alelos com efeitos aditivos em ambientes com solos ácidos (Tabela 2).

A contribuição dos efeitos de dominância (DLI) em decorrência da seleção foi negativa $\left(-3,06 \times 10^{-2}\right.$ dias $)$ e significativa $(\mathrm{P} \leq 0,05)$ no caráter florescimento feminino, no processo de seleção combinada. Os efeitos aditivos (ALI) contribuíram positiva e não significativamente $\left(0,52 \times 10^{-2}\right.$ dias $)$, indicando a preponderância dos efeitos de dominância em diminuir os dias para florescimento feminino. Por outro lado, no processo de meios-irmãos modificados, os efeitos de dominância em virtude da seleção foram negativos $\left(-0,92 \times 10^{-1}\right.$ dias $)$ e significativos $(\mathrm{P} \leq 0,01)$, e as estimativas dos efeitos aditivos foram positivas $\left(0,48 \times 10^{-1}\right.$ dias $)$ e significativas $(\mathrm{P} \leq 0,01)$, embora de menor magnitude. No processo de irmãos germanos, as estimativas foram negativas e não-significativas, apresentando magnitudes de $-0,52 \times 10^{-1}$ dias em relação aos efeitos aditivos e de $-0,64 \times 10^{-1}$ dias em relação aos efeitos de dominância.

A estimativa da perda de heterozigose decorrente da oscilação genética (DQI) foi positiva $\left(0,61 \times 10^{-3}\right.$ dias $)$ e não-significativa na seleção combinada; no caso de meios-irmãos modificados, foi positiva $\left(0,32 \times 10^{-2}\right.$ dias $)$ e significativa $(\mathrm{P} \leq 0,01)$, e no caso de irmãos germanos foi negativa $\left(-0,37 \times 10^{-1}\right.$ dias $)$ e significativa $(P \leq 0,10)$. Esses resultados mostram que a oscilação genética apresentou uma tendência inconsistente em magnitude e

TABELA 3. Estimativas da resposta esperada à seleção (2ALI+2DLI) na população SA3, em três processos seletivos e quatro caracteres agronômicos em ambientes com solos ácidos (1995-1997).

\begin{tabular}{lcccc}
\hline Processo seletivo $^{1}$ & $\begin{array}{c}\text { Produção de grãos } \\
\left(\mathrm{t} \mathrm{ha}^{-1}\right)\end{array}$ & $\begin{array}{c}\text { Florescimento feminino } \\
(\text { dias })\end{array}$ & $\begin{array}{c}\text { Altura da planta } \\
(\mathrm{cm})\end{array}$ & $\begin{array}{c}\text { Número de espigas } \\
\text { por planta }\end{array}$ \\
\hline${\mathrm{MIM}-\mathrm{IG}^{2}}^{0,72 * * * \pm 0,39}$ & $-5,08^{* *} \pm 1,11$ & $2,64^{\mathrm{ns}} \pm 7,60$ & $0,16^{* * *} \pm 0,09$ \\
$\mathrm{MIM}^{3}$ & $0,22^{* *} \pm 0,06$ & $-0,88^{* *} \pm 0,17$ & $0,26^{\mathrm{ns}} \pm 1,52$ & $0,018^{\mathrm{ns}} \pm 0,014$ \\
$\mathrm{IG}^{3}$ & $0,28^{\mathrm{ns}} \pm 0,37$ & $-2,32^{*} \pm 1,05$ & $0,42^{\mathrm{ns}} \pm 7,22$ & $0,18^{* * *} \pm 0,09$ \\
\hline
\end{tabular}

${ }^{1}$ Meios-irmãos modificados e irmãos germanos (MIM-IG); meios-irmãos modificados (MIM); irmãos germanos (IG).

2 (2ALI+2DLI) $\left(10^{-1}\right)$.

3 (2ALI+2DLI) $\left(10^{-2}\right)$.

$*, * *$ e *** Significativo a $5 \%, 1 \%$ e $10 \%$, respectivamente. 
sinal com os processos seletivos. No entanto, para o processo de irmãos germanos, DQI foi negativo e significativo e, portanto, contribuiu para reduzir o número de ciclos para o florescimento (Tabela 2).

Em relação à resposta esperada à seleção $(2 \mathrm{ALI}+2 \mathrm{DLI})$ nos três processos seletivos, os resultados foram de $-5,08 \times 10^{-2}$ dias $(-0,09 \%)$; $-0,88 \times 10^{-1}$ dias $(-0,13 \%)$ e $-2,32 \times 10^{-1} \operatorname{dias}(-0,36 \%)$ na seleção combinada, de meios-irmãos modificados e de irmãos germanos, respectivamente. A estimativa da resposta esperada à seleção nesta pesquisa quanto a esse caráter foi diferente em magnitude, porém apresentou a mesma direção daquela relatada por Granados et al. (1993) nessa população (Tabela 3). Em termos gerais, os resultados obtidos quanto a esse caráter em condições de solos ácidos, indicam que o processo de seleção recorrente intrapopulacional foi eficiente para reduzir os dias para florescimento feminino nessa população. Ressalta-se que a resposta à seleção com progênies de irmãos germanos foi superior à resposta com progênies de meios-irmãos. Isso já era esperado, uma vez que no processo de irmãos germanos aproveita-se uma maior quantidade da variância genética aditiva que no processo de meios-irmãos.

No caráter altura da planta, as estimativas dos efeitos aditivos (AOI) e de dominância (DOI) na população original foram positivas e significativas $(\mathrm{P} \leq 0,01)$ nos três processos seletivos, apresentando magnitudes de 138,82 e $14,75 \mathrm{~cm}$ no processo de seleção combinada; de 139,83 e de 13,83 cm no processo de meios-irmãos modificados, e de 150,68 e $10,08 \mathrm{~cm}$ para irmãos germanos, respectivamente, indicando predominância dos efeitos aditivos nos três processos seletivos (Tabela 2). Os efeitos aditivos (ALI) resultantes da seleção contribuíram positiva $\left(13,39 \times 10^{-2} \mathrm{~cm}\right)$ e significativamente $(\mathrm{P} \leq 0,10)$ na seleção combinada; positiva $\left(0,97 \times 10^{-1} \mathrm{~cm}\right)$, e significativamente $(\mathrm{P} \leq 0,10)$ para meios-irmãos modificados, e negativa $\left(-6,16 \times 10^{-1} \mathrm{~cm}\right)$ e não-significativamente para irmãos germanos. Entretanto, as estimativas dos efeitos de dominância decorrentes da seleção (DLI) não foram significativas nos três processos seletivos, sendo negativas na seleção combinada $\left(-12,07 \times 10^{-1} \mathrm{~cm}\right)$ e meios-irmãos modificados $\left(-0,84 \times 10^{-1} \mathrm{~cm}\right)$ e positiva $\left(6,37 \times 10^{-1} \mathrm{~cm}\right)$ no processo de irmãos germanos. As estimativas dos efeitos aditivos e dominantes relativas a esse caráter apresentaram uma tendência inconsistente em magnitude e sinal. E as estimativas dos efeitos causados pela oscilação genética (DQI), foram positivas $\left(0,26 \times 10^{-3} \mathrm{~cm}\right.$ e $\left.0,92 \times 10^{-2} \mathrm{~cm}\right)$ e não-significativas nos processos de seleção combinada e de meiosirmãos modificados, respectivamente; sendo negativas $\left(-2,24 \times 10^{-1}\right)$ e significativas $(\mathrm{P} \leq 0,10)$ no processo de irmãos germanos. Cabe salientar que as estimativas da oscilação genética no processo de irmãos germanos foram no sentido oposto à direção da resposta à seleção, apresentando tendência a limitar o melhoramento da população SA3 (Tabela 2). As respostas esperadas à seleção $(2 \mathrm{ALI}+2 \mathrm{DLI})$ apresentaram estimativas de 2,64 x 10-2 $\mathrm{cm}(0,01 \%)$ na seleção combinada; $0,26 \times 10^{-1} \mathrm{~cm}(0,01 \%)$ para meios-irmãos modificados, e $0,42 \times 10^{-1} \mathrm{~cm}(0,02 \%)$ para irmãos germanos (Tabela 3 ). As estimativas da resposta à seleção são bastante inferiores às relatadas na população SA3 sob condições de solos ácidos (Granados et al., 1993). Essas diferenças poderiam ser atribuídas ao número de ambientes de avaliação e dos diferentes níveis de saturação de $\mathrm{Al}$ presente nesses ambientes. As estimativas da resposta à seleção para altura da planta mostram que os processos de seleção utilizados não foram eficientes para melhorá-los em solos ácidos, uma vez que ocorreram aumentos nesse caráter, o que é indesejável.

Quanto ao número de espigas por planta, os efeitos aditivos (AOI) e de dominância (DOI) contribuíram positiva e significativamente $(\mathrm{P} \leq 0,01)$ nos três processos seletivos, indicando, assim, que ambos os tipos de efeitos foram importantes na população original. As magnitudes dessas estimativas foram de 0,59 e 0,17 espigas por planta no processo de seleção combinada, de 0,57 e 0,18 espigas por planta no processo de meios-irmãos modificados, e de 0,54 e 0,20 espigas por planta em irmãos germanos, evidenciando preponderância dos efeitos aditivos sobre os de dominância. Os efeitos aditivos (ALI) decorrentes da seleção foram positivos e não-significativos na seleção combinada e no processo de meiosirmãos modificados, apresentando magnitudes de $0,09 \times 10^{-2}$ e $0,01 \times 10^{-1}$ espigas por planta, respectivamente. No processo de irmãos germanos, esse efeito foi positivo $\left(0,21 \times 10^{-1}\right.$ espigas por planta) e significativo $(\mathrm{P} \leq 0,10)$. Por outro lado, as estimativas 
dos efeitos de dominância (DLI) decorrentes da seleção foram negativas, não-significativas, e de menor magnitude nos três processos seletivos $\left(-0,008 \times 10^{-2},-0,008 \times 10^{-1} \mathrm{e}-0,12 \times 10^{-1}\right.$ espigas por planta, respectivamente). Esses resultados mostram que nesse caráter os efeitos aditivos são mais importantes que os efeitos de dominância, como relatados para diferentes populações em solos ácidos (DuqueVargas et al., 1994; Borrero et al., 1995; Resende et al., 1997). No que se refere às estimativas da perda de heterozigose por causa da oscilação genética (DQI), estas apresentaram-se positivas e não-significativas, e portanto, não limitam as respostas à seleção. A resposta à seleção, nos três processos seletivos, apresentou as seguintes magnitudes: de $0,16 \times 10^{-2}$ espigas por planta $(0,17 \%)$ na seleção combinada; de $0,018 \times 10^{-1}$ espigas por planta $(0,19 \%)$ no processo de meios-irmãos modificados, e de $0,18 \times 10^{-1}$ espigas por planta $(1,91 \%)$ no caso de irmãos germanos (Tabela 3). Esses resultados mostram que o processo de seleção utilizado foi eficiente para melhorar esse caráter, e que os maiores progressos com seleção foram obtidos no processo de irmãos germanos.

Ressalte-se que, no caso da produção de grãos, que é o caráter mais importante, os efeitos da oscilação genética (DQI) não foram significativos, e as respostas à seleção no processo da seleção combinada (MIM-IG) e na seleção com meios-irmãos modificados foram positivos e significativos, embora de baixa magnitude, o que não ocorreu com o processo de irmãos germanos. O fato de DQI não ser significativo deve-se ao elevado tamanho efetivo populacional mantido durante os ciclos seletivos, embora o fato de o processo seletivo com progênies de irmãos germanos não apresentar resposta à seleção não seja evidente, uma vez que teoricamente espera-se que esse processo seja mais eficiente que o processo de meios-irmãos modificados. Assim, para que o melhoramento dessa população possa ser feito de forma eficiente seria necessário alterar o processo seletivo e as condições experimentais para a avaliação das progênies visando minimizar a elevada heterogeneidade desse tipo de solo.

\section{CONCLUSÕES}

1. Os processos seletivos utilizados apresentam baixa eficiência no melhoramento dos caracteres produção de grãos e altura de planta e são eficientes para os caracteres dias para o florescimento feminino e número de espigas por planta sob condições de solos ácidos.

2. As estimativas da oscilação genética mostram que seus efeitos não limitam o melhoramento dos caracteres avaliados.

\section{AGRADECIMENTOS}

À Fundação de Amparo à Pesquisa do Estado de São Paulo (FAPESP) e ao Centro Internacional de Mejoramiento de Maíz y Trigo (CIMMYT), pela concessão de bolsa de estudos e apoio financeiro.

\section{REFERÊNCIAS}

AGUIAR, P.A. Avaliação de progênies de meios-irmãos da população de milho CMS-39 em diferentes condições de ambiente. Lavras : UFLA, 1986. 69p. Dissertação de Mestrado.

BORRERO, J.C.; PANDEY, S.; CEBALLOS, H.; MAGNAVACA, R.; BAHIA FILHO, A.F.C. Genetic variances for tolerance to soil acidity in a tropical maize population. Maydica, Bergamo, v.40, p.283288, Apr./June 1995.

COCHRAN, G.W.; COX, M.G. Experimental designs. 2.ed. New York : J. Wiley, 1957. 611p.

DUQUE-VARGAS, J.; PANDEY, S.; GRANADOS, G.; CEBALLOS, H.; KNAPP, R. Inheritance of tolerant to soil acidity in tropical maize. Crop Science, Madison, v.34, p.50-54, Jan./Feb. 1994.

EBERHART, S.A. Least squares method for comparing progress among recurrent selection methods. Crop Science, Madison, v.4, p.230-231, Mar./Apr. 1964.

GRANADOS, G.; PANDEY, S.; CEBALLOS, H. Response to selection for tolerance to acid soils in a tropical maize population. Crop Science, Madison, v.33, p.936-940, Sept./Oct. 1993.

HALLAUER, A.R.; MIRANDA FILHO, J.B. Quantitative genetics in maize breeding. Ames : Iowa State University Press, 1981. 468p.

HELMS, T.C.; HALLAUER, A.R.; SMITH, S.O. Genetic variability estimates in improved and non improved 'Iowa stiff stalk synthetic' maize populations. Crop Science, Madison, v.29, p.959-962, July/Aug. 1989.

IGLESIAS, C.A.; HALLAUER, A.R. S recurrent selection in maize populations with exotic germplasm. Maydica, Bergamo, v.34, p.133-140, Jan./Mar. 1989. 
KEERATINIJAKAL, V.; LAMKEY, R.K. Genetic effects associated with reciprocal recurrent selection in BSSS and BSCB1 maize populations. Crop Science, Madison, v.33, p.78-82, Jan./Feb. 1993a.

KEERATINIJAKAL, V.; LAMKEY, K.R. Responses to reciprocal recurrent selection in BSSS and BSCB1 maize populations. Crop Science, Madison, v.33, p.73-77, Jan./Feb. 1993b.

LIMA, M.; FURLANI, P.R.; MIRANDA FILHO, J.B. de. Divergent selection for aluminum tolerance in a maize (Zea mays L.) population. Maydica, Bergamo, v.37, p.123-132, Jan./Mar. 1992.

LOPES, M.A.; MAGNAVACA, R.; BAHIA FILHO, A.F.C.; GAMA, E.E.G. Avaliação de populações de milho e seus cruzamentos para tolerância à toxidez de alumínio em solução nutritiva. Pesquisa Agropecuária Brasileira, Brasília, v.22, n.3, p.257-263, mar. 1987.

PANDEY, S.; CEBALLOS, H.; KNAPP, E.B.; VARGAS, J.C. Genetic variability in maize for adaptation to acid soil. In: SIMPÓSIO INTERNACIONAL SOBRE ESTRESSE AMBIENTAL, 1992, Sete Lagoas. Anais. Sete Lagoas : Embrapa-CNPMS/CIMMYT/ United Nations Development Program, 1995. p.295-319.

PARENTONI, S.N.; BAHIA FILHO, A.F.C.; GAMA, E.E.G.; LOPES, M.A.; GUIMARÃES, P.E.O.; SANTOS, M.X. Capacidade combinatória de linhagens elites de milho em solos ácido e fértil. In: REUNIÓN LATINOAMERICANO, 3., REUNIÓN DE LA ZONA ANDINA DE INVESTIGADORES EN MAIZ, 16., 1995, Cochabamba. Memórias. Cochabamba : Centro de Investigaciones Fitoecogenéticas de Pairumani, 1995. p.35-46.

PARENTONI, S.N.; GAMA, E.E.G.; LOPES, M.A.; SANTOS, M.X.; OLIVEIRA, P.E.; ELEUTÉRIO, A.; BAHIA FILHO, A.F.C.; VERA, M.C.A.; FRANÇA, G.E.C.; COELHO, A.M.; CORREA, L.A.; PAIVA, E.; VASCONCELOS, M.J.V. Avanços no programa de adaptação de milho a solos ácidos do CNPMS/EMBRAPA, 1994-1996. In: REUNIÓN DE COORDINADORES SURAMERICANOS DE PROGRAMAS DE MAIZ, 4., 1996, Cali. Memorias. Cali : CIMMYT-Programa Suramericano de Maíz, 1996. p.74-103.

PIMENTEL-GOMES, F.; GARCIA, C.H. Experimentos em látice: planejamento e análise por meio de «pacotes» estatísticos. Piracicaba : Instituto de Pesquisas e Estudos Florestais/ESALQ, 1991. 69p.
RESENDE, M.D.V. de; SOUZA JUNIOR, C.L. de; GAMA, E.E.G.; MAGNAVACA, R. Análise quantitativa da seleção envolvendo progênies de milho (Zea mays L.) em solos sob cerrado e fértil. I. Progressos genéticos. Pesquisa Agropecuária Brasileira, Brasília, v.32, n.5, p.495-507, maio 1997.

REZENDE, G.D.S.P. Heterose, depressão por endogamia e variabilidade genética associadas à seleção e oscilação genética nas populações de milho BR-105 e BR-106. Piracicaba : ESALQ, 1997. 112p. Tese de Doutorado.

SALAZAR, F.S.; PANDEY, S.; NARRO, L.; PEREZ, J.C.; CEBALLOS, H.; PARENTONI, S.N.; BAHIA FILHO, A.F.C. Diallel analysis of acid-soil tolerant and intolerant tropical maize populations. Crop Science, Madison, v.37, p.1457-1462, Sept./Oct. 1997.

SCAPIM, C.A.; CARVALHO, C.G.P. de; CRUZ, C.M. Uma proposta de classificação dos coeficientes de variação para a cultura de milho. Pesquisa Agropecuária Brasileira, Brasília, v.30, n.5, p.683686, maio 1995.

SMITH, O.S. Application of a modified diallel analysis to evaluate recurrent selection for grain yield in maize. Crop Science, Madison, v.19, p.819-822, Nov./Dec. 1979a.

SMITH, O.S. Evaluation of recurrent selection in BSSS, BSCB1, and BS13 maize populations. Crop Science, Madison, v.23, p.35-40, Jan./Feb. 1983.

SMITH, O.S. A model for evaluating progress from recurrent selection. Crop Science, Madison, v.19, p.223-226, Mar./Apr. 1979b.

SOUZA JUNIOR, C.L. de. Variabilidade genética em milho (Zea mays L.) e relações com a seleção recorrente intra e interpopulacional. Piracicaba : ESALQ, 1983. 151p. Tese de Doutorado.

STEEL, R.G.D.; TORRIE, J.H. Principles and procedures of statistics. New York: McGraw-Hill, 1960. $481 \mathrm{p}$.

STOJSIN, D.; KANNENBERG, L.W. Changes associated with different methods of recurrent selection in five maize populations. II. Indirectly selected traits. Crop Science, Madison, v.34, p.1473-1479, Nov./Dec. 1994a.

STOJSIN, D.; KANNENBERG, L.W. Genetic changes associated with different methods of recurrent selection in five maize populations. I. Directly selected traits. Crop Science, Madison, v.34, p.1466-1472, Nov./Dec. 1994b.

Pesq. agropec. bras., Brasília, v.35, n.8, p.1585-1593, ago. 2000 